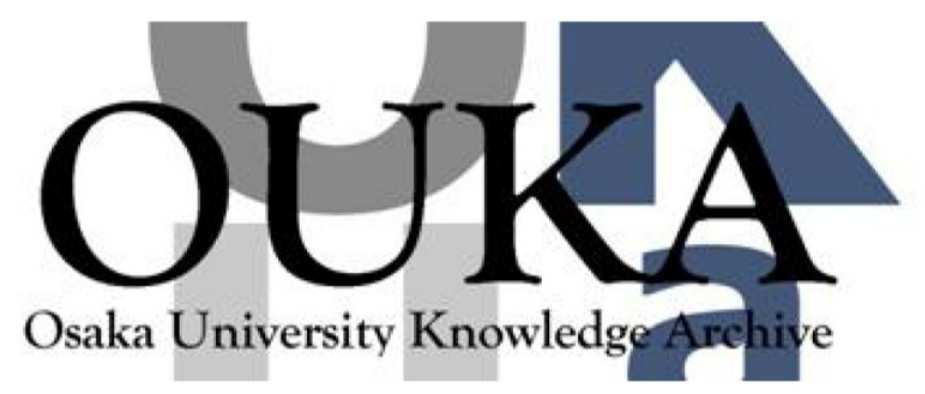

\begin{tabular}{|c|l|}
\hline Title & $\begin{array}{l}\text { Bimodal fingerprint capturing system based on } \\
\text { compound-eye imaging module }\end{array}$ \\
\hline Author(s) & $\begin{array}{l}\text { Shogenji, Rui; Kitamura, Yoshiro; Yamada, Kenj i } \\
\text { et al. }\end{array}$ \\
\hline Citation & Applied Optics. 43(6) p. 1355-p. 1359 \\
\hline Issue Date & 2004-02-20 \\
\hline oaire:version & VoR \\
\hline URL & https://hdl. handle.net/11094/3224 \\
\hline rights & \\
\hline Note & \\
\hline
\end{tabular}

Osaka University Knowledge Archive : OUKA

https://ir. Library. osaka-u. ac. jp/

Osaka University 


\title{
Bimodal fingerprint capturing system based on compound-eye imaging module
}

\author{
Rui Shogenji, Yoshiro Kitamura, Kenji Yamada, Shigehiro Miyatake, and Jun Tanida
}

\begin{abstract}
A compact imaging system called TOMBO (Thin Observation Module by Bound Optics) is proposed in which a microlens array is used for thin hardware configuration. This paper describes a fingerprintcapturing module as an application of the TOMBO. Experimental results by the TOMBO prototype system are shown to clarify the applicability of the TOMBO to the fingerprint capturing. Different types of biometrics, i.e., fingerprint and face images, are captured by the same hardware, which shows the extendability of the system for multimodal identification. (C) 2004 Optical Society of America
\end{abstract}

OCIS codes: $110.2970,110.4190,100.3020,230.0250,230.3120$.

\section{Introduction}

In recent years, authentication has been an important issue for the realization of various services over the Internet, such as electronic commerce or electronic government. ${ }^{1}$ Biometrics techniques using the information of a living body like a fingerprint or an iris are promising, because they can improve security strength as compared with conventional methods using a password or a personal identification number. ${ }^{2}$ One application of the authentication is found in electronic commerce using a personal digital assistant, such as a cellular phone. In such a system, the identification of the legal user is crucial to the security of the system. For this purpose, a personal digital assistance device with the functionality of authentication is considered to be as effective. ${ }^{3}$

Among various biometrics, fingerprint identification has been studied actively owing to its convenient usage and effective performance. ${ }^{4}$ A key issue of the fingerprint identification is how to obtain fingerprint data so that various types of fingerprint sensors using

R. Shogenji (rui@photonics.mls.eng.osaka-u.ac.jp) and Y. Kitamura are with the Graduate School of Engineering, Osaka University, 2-1 Yamadaoka, Suita, Osaka 565-0871, Japan. K. Yamada is with Innovation Plaza Osaka, Japan Science and Technology Corporation, 3-1-10 Techno-Stage Izumi, Osaka 594-1144, Japan. S. Miyatake is with the Minolta Company, Ltd., 1-2 Sakura-machi, Takatsuki, Osaka 569-5803, Japan. J. Tanida is with the Graduate School of Science and Technology, Osaka University, 2-1 Yamadaoka Suita, Osaka 565-0871, Japan.

Received 26 May 2003; revised manuscript received 29 October 2003; accepted 5 November 2003.

0003-6935/04/061355-05\$15.00/0

(C) 2004 Optical Society of America optical imaging or electric capacitive distribution are investigated. The TOMBO (Thin Observation Module by Bound Optics) system, a compound-eye imaging system proposed by the authors, ${ }^{5}$ is attractive for the fingerprint sensor because of its thin hardware configuration. However, the TOMBO system was originally designed to capture the image of an object located at a distant position. Therefore, by modification of the optical system, we adapt the TOMBO system to capture the image of an object at a near position.

In this paper, an application of the TOMBO system to fingerprint capturing is presented. In Section 2 the principle of fingerprint capturing based on the TOMBO system is explained. In Section 3 an experimental result by the TOMBO prototype system is described. In Section 4 bimodal image capturing, in which fingerprint and face images are captured by the same hardware, is demonstrated as an interesting extension of the TOMBO system. The result provides a notable feature of the TOMBO architecture, which is expected to be useful for multimodal authentication.

\section{Fingerprint Capturing by Use of the TOMBO}

A fundamental configuration of the TOMBO is shown in Fig. 1. The TOMBO is composed of a microlens array, a signal separator, and a photodetector array. A signal separator prevents optical signal cross talk between the adjacent lenses. The TOMBO provides small and lightweight hardware through miniaturization of the lenses. Usually, miniaturization of the optical system causes a reduction of the image resolution. However, in the TOMBO system, signal processing retrieves a high-resolution image using 


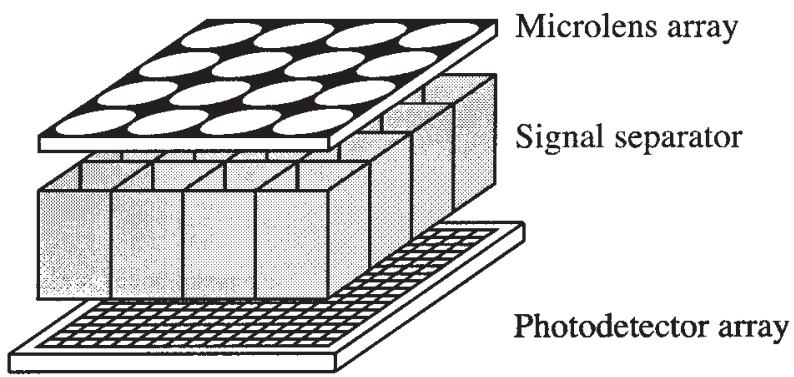

Fig. 1. TOMBO system configuration.

multiple low-resolution images captured by compact imaging systems. When the low-resolution images have different sampling information, they can be utilized for the image reconstruction. For use as the reconstruction method, the image-sampling method, ${ }^{5}$ the backprojection method, ${ }^{5}$ and the pixel-rearrange method $^{6}$ were proposed. The experimental results by use of the TOMBO prototype system show the effectiveness of the pixel-rearrange method.

The fingerprint-capturing system based on the TOMBO is composed of the same elements as the conventional TOMBO system, except for the illumination components. The original TOMBO system is set to focus on infinity or a near-infinity point. On the other hand, the proposed TOMBO system for fingerprint capturing is set to focus on the surface of the light-guide plate.

Figure 2 shows a setup of the fingerprint-capturing module. An elemental imaging system corresponding to a microlens is called a unit. An image captured by a unit is called a unit image. A set of unit images captured by the entire system is called a compound image. In the original TOMBO system, all the units observe almost the same part of the target at a time. On the other hand, the proposed system observes a different part on a unit-by-unit basis. The captured compound image is a set of rotated images captured by each unit. The wall of the signal

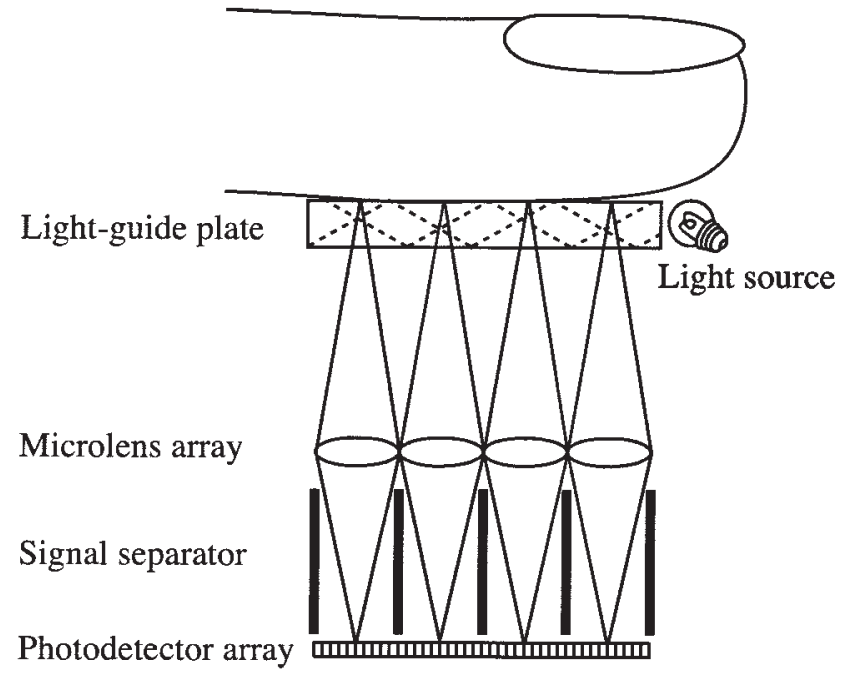

Fig. 2. Setup of the TOMBO system for fingerprint capturing. separator obstructs the incident light, so that the edge of the unit image becomes dark. Therefore the magnification ratio of the optical system is set as less than unity in order to not lose the information of the fingerprint. The magnification ratio of the optical system $m$ for the maximum resolution of the fingerprint image is provided by

$$
m=1-\frac{2 p}{l}\left\lceil\frac{w}{2 p}\right\rceil,
$$

where $l, w$, and $p$ are the lens pitch, the thickness of the separation wall, and the pixel pitch of the imaging sensor, respectively. \lceil\rceil means a roundup operation below the decimal point.

Instead of the reconstruction methods developed for the TOMBO system, the following procedure is adopted to achieve simple and fast reconstruction.

1. Shading compensation and fixed pattern noise reduction.

2. Separation of the unit images.

3. Removal of the edge pixels in the unit image.

4. Rotation of the clipped unit image.

5. Combination of the all rotated images.

6. Gray level correction.

For the preprocessing, the shading and the fixed pattern noise are compensated with a white reference image and the background image. The captured compound image is divided into the unit images. Based on the magnification ratio of the optical system, the edge pixels in the unit image are removed. The clipped unit image is rotated $180^{\circ}$, and all of the processed images are combined. The gray level of the resulting image is corrected to enlarge the dynamic range. Finally a segment image of the fingerprint is obtained.

The field-view size of the fingerprint is limited by the chip area of the image sensor. However, this is a simple restriction of available chip, and a large area chip can enlarge the field view with an increase of the fabrication cost. Another option is to tile multiple small sensors for emulation of a large sensor. Instead, we use a novel method in which multiple fingerprint images at different points are captured by moving the finger on the sensor. All of the segment images are combined to reconstruct a large area of the fingerprint. The relative distance between the two images is calculated by the pattern-matching method. If sequential detection is introduced, not only the shape of the fingerprint but also the action of the finger can be detected by the same sensor.

The image combining is achieved by the following procedure. The target fingerprint is assumed to be moved relative to the sensor, and a sequence of the segment images of the fingerprint is captured. Let $f(x, y)$ and $g(x, y)$ be two successive segment images through movement of the finger, each of which is reconstructed from the captured data. The distance between the two segment images is estimated by the pattern matching method. The sequential similar- 
ity detection algorithm is used for the pattern matching. ${ }^{7}$ In this algorithm, the fingerprint image is binarized by a threshold operation used as preprocessing. The summation of the absolute value of the difference between the overlapped pixels is calculated with scanning the image $g(x, y)$ over the reference image $f(x, y)$. The normalized value by the number of the overlapped pixels is defined as the degree of nonsimilarity $D$.
It is difficult to measure exact microlensphotodetector distance because of the difficulty in determining the principal points of the microlenses. Therefore we presumably set the object-microlens distance and the trimming size as $32 \times 32$ pixels for reconstruction. In this case, the object distance and the magnification ratio of the system are $5.46 \mathrm{~mm}$ and 0.64 , respectively. Then we adjust the microlensphotodetector distance to obtain a smooth connection

$$
D(u, v)=\frac{\sum_{j=0}^{h-|v|} \sum_{i=0}^{w-|u|}\left|f\left[\frac{i+(u+|u|)}{2}, \frac{j+\overline{(v+|v|)}}{2}\right]-g\left[\frac{i+(u-|u|)}{2}, \frac{j+(v-|v|)}{2}\right]\right|}{(h-|v|)(w-|u|)},
$$

where $u, v, w$, and $h$ are the horizontal scanning distance, the vertical scanning distance, the segment image width, and the segment image height, respectively.

The position $(u, v)$, which has the minimum degree of nonsimilarity, provides the moving distance between two images. The image $g(x, y)$ is used as the reference image $f(x, y)$ for the next step. The same process is repeated for all the segment images. Finally, these segment images are combined by use of the estimated moving distances.

\section{Experiment}

\section{A. System Setup}

To study the performance of the presented method, we captured a fingerprint image using the TOMBO prototype system. The specifications of the microlens array (Advanced Microoptic Systems GmbH, Saarbruecken, Germany, APO-Q-P500-AF1.3) are: lens pitch, $500 \mu \mathrm{m}$; focal length of the element lens, $1.3 \mathrm{~mm}$; and diameter of the element lens, $500 \mu \mathrm{m}$. The signal separator is made by thermocompression of 21 sheets of stainless steel plates $(50-\mu \mathrm{m}$ thickness), whose holes are fabricated by etching process. The surface of the etched hole is modified by antireflection coating. The thickness and the height of the signal separator are approximately 50 and $1050 \mu \mathrm{m}$, respectively. The wall pitch is $500 \mu \mathrm{m}$, which is equal to the lens pitch. For the image sensor, a custom-designed complementary metal oxide semiconductor photodetector array (austriamicrosystems $0.6 \mu \mathrm{m}$ ) is used. The number of pixels is $604 \times 504$, and the pixel pitch is $10.0 \mu \mathrm{m}$. Fifty pixels of the left and of the right sides are used for lens alignment and dark current calibration. The number of pixels per unit is $50 \times 50$. Up to $10 \times 10$ units can be used for the reconstruction processing.

The microlens array is held by a six-axis stage and set in front of the image sensor. A translucent object illuminated by white light from the back side is used instead of an actual fingerprint, owing to lack of the illumination components. For the object a printed fingerprint image (SIDBA FINGERPRNT08) is used. over the reconstructed image. The optimum position is decided by visual inspection.

\section{B. Experimental Results}

The captured image by the experimental TOMBO system is shown in Fig. 3 . The image size is $500 \times$ 500 pixels. The captured image is compensated with respects to the shading and the fixed pattern noise by use of the reference images captured previously.

The reconstructed fingerprint image with the trimming size of $32 \times 32$ pixels is shown in Fig. 4 . The image size is $320 \times 320$ pixels. The observation area is $5 \times 5 \mathrm{~mm}^{2}$, which is determined by the lens pitch and the number of units. The resolution of the fingerprint image is 1727.2 dots per inch (dpi).

The four pieces of the segment images are captured by changing the observation position of the target. The moved distance between the segment images are estimated, and these values are used to connect them as shown in Fig. 5. As seen from the figure, each segment image is combined correctly, and the reconstructed image of $12.8 \times 5.0 \mathrm{~mm}^{2}$ is retrieved.

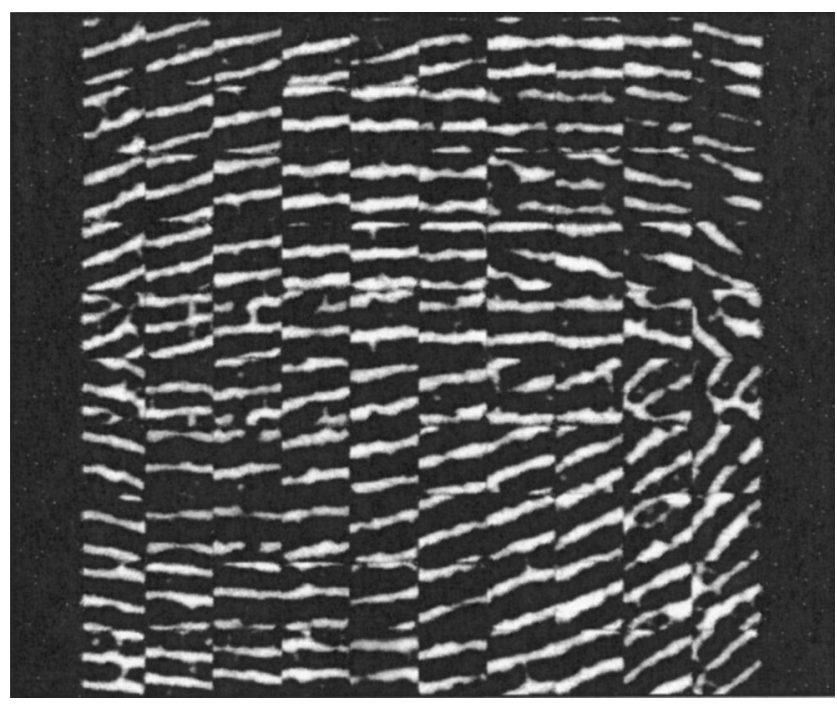

Fig. 3. Captured images. 


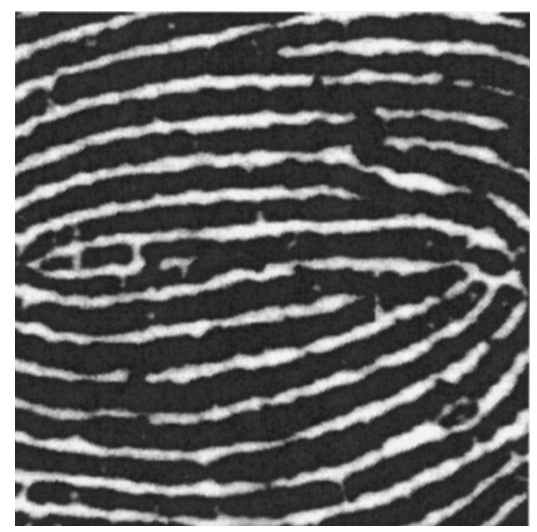

Fig. 4. Retrieved fingerprint images.

\section{Discussion}

The thickness of the system depends mainly on the focal length of the microlens and the magnification ratio of the optical system. The magnification ratio of the optical system $m$ is determined by

$$
m=\frac{2 p}{l}\left\lceil\frac{l r}{50.8 \times 10^{3}}\right\rceil,
$$

where the required resolution of the fingerprint image, the lens pitch, and the pixel pitch are $r$ dpi, $l \mu \mathrm{m}$, and $p \mu \mathrm{m}$, respectively.

Figure 6 shows the calculation result of the relationship between the object distance and the resolution of the fingerprint image when the lens pitch, the focal length, and the pixel pitch are $500 \mu \mathrm{m}, 1.3 \mathrm{~mm}$, and $10 \mu \mathrm{m}$, respectively. The resolution for scanning a fingerprint is typically $500 \mathrm{dpi},{ }^{8}$ so the object distance of the system should be set near $6-8 \mathrm{~mm}$.

The proposed system can capture a high-resolution fingerprint image. On the other hand, if the magnification ratio is set small and if the lensphotodetector distance is close to the focal length of the lens, the same hardware can capture an object located at a distant position as well as an object on the surface of the lens. For example, we can capture both the fingerprint and the face images with the same hardware. However, with the original configuration for the fingerprint capturing, the captured

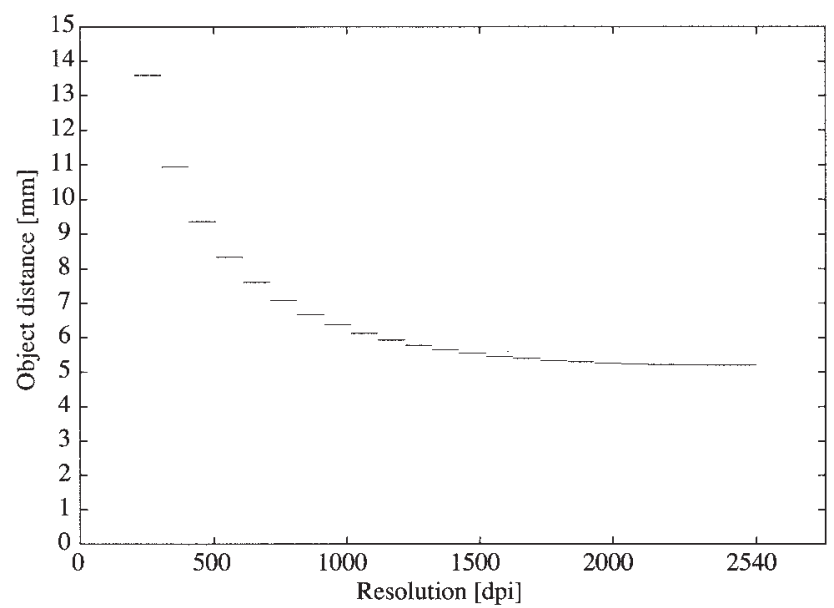

Fig. 6. Relationship between the object distance and the resolution of the fingerprint image. The lens pitch, the focal length, and the pixel pitch are $500 \mu \mathrm{m}, 1.3 \mathrm{~mm}$, and $10 \mu \mathrm{m}$, respectively.

image of the face is remarkably out of focus. A solution of this problem, a focus adjustment mechanism that uses a microactuator such as a piezoelectric actuator, can be considered. However, the compactness of the TOMBO system is greatly reduced by the mechanism. Therefore as another solution we design an optical system balancing the defocus of the both imaging modes.

The same specifications of the microlens as used in the experimental system are assumed. The required resolution of the fingerprint image is set as $500 \mathrm{dpi}$. The trimming size is set to $10 \times 10$ pixels from the resolution. The amount of the defocus is set to the distance where both the diameter of the blur circle in the face image and the fingerprint image-capturing mode are almost equal. The diameter of blur circle $\delta$ for the face image-capturing mode is provided by

$$
\delta=\frac{b}{a} \epsilon,
$$

where $a, b$, and $\epsilon$ are the lens-photodetector distance, the diameter of the element lens, and the amount of defocus, respectively. In the same way, the diame-

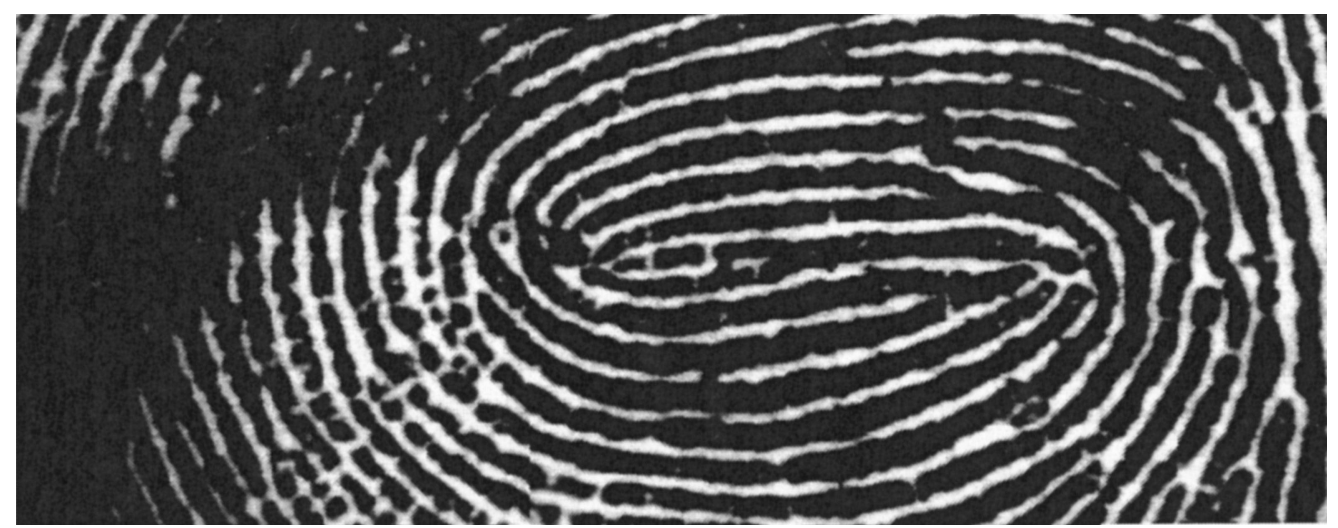

Fig. 5. Combined fingerprint images. 


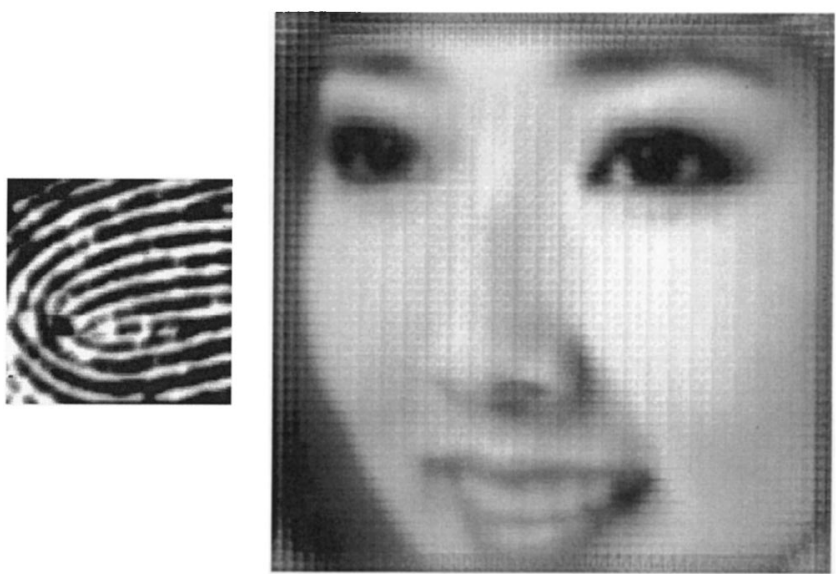

Fig. 7. Captured images of the fingerprint and the face by use of the same TOMBO system.

ter of blur circle $\delta^{\prime}$ for the fingerprint-capturing mode is provided by

$$
\delta^{\prime}=\frac{b}{a^{\prime}} \epsilon^{\prime},
$$

where $a^{\prime}$ and $\epsilon^{\prime}$ are the lens-photodetector distance and the amount of defocus, respectively.

When the trimming size was assumed to be $10 \times 10$ pixels, the optimal lens-photodetector distance is $1.56 \mathrm{~mm}$ for the fingerprint-capturing mode. Also, the optimal lens-photodetector distance for the face image-capturing mode is $1.3 \mathrm{~mm}$. In our experiment, the lens-photodetector distance is set to 1.41 $\mathrm{mm}$, introducing a $150-\mu \mathrm{m}$ defocus for the fingerprint-capturing mode. Therefore the amount of the defocus for the face image becomes $110 \mu \mathrm{m}$. In this case, the diameter of the blur circle for the face image and the fingerprint-capturing mode are approximately 42.30 and $48.07 \mu \mathrm{m}$, respectively. Since these values are almost equal, we took this setup.

Figure 7 shows the captured images of the fingerprint and the face by use of this alignment. The object distance and the captured area are approximately 325 and $125 \times 125 \mathrm{~mm}^{2}$, respectively. Both reconstructed images are sharpened with a digital filter. Although these images are slightly out of focus, they have sufficient information for personal authentication, such as the fingerprint minutia and features of the face. This experimental result shows a capability of the proposed TOMBO system for the bimodal image capturing of fingerprint and face images with the same hardware. This technique can be utilized for multimodal identification, in which fingerprint verification and face recognition are implemented with a single compact hardware.

In the proposed method, finger movement can be incorporated into the verification process on the basis of the fingerprint pattern to improve recognition accuracy. Finger movement is tracked by connecting the multiple segment images with the pattern matching method. The speed and the direction of the finger movement is also detected through sequential analysis of the segment images. Therefore a series of finger movements can be used as a personal identification key. In addition, finger action is utilized as an input method for a device with authentication capability.

\section{Summary}

A thin fingerprint-capturing module using the compound-eye imaging system TOMBO has been proposed. A simple and high-speed reconstruction method for the fingerprint capturing has been described. A fingerprint image of 1727.2 dpi was captured by the experimental system. The experimental result proved the capability of capturing a fingerprint image by the proposed method. A large area of an image can be successfully reconstructed by a combination of multiple segment fingerprint images with the pattern-matching method. As a notable feature of this system, fingerprint and face images can be captured with the same hardware. With these functionalities, the proposed system is expected to be applied to a multimodal authentication system.

This research was supported by Development of Basic Tera Optical Information Technologies, Osaka Prefecture Collaboration of Regional Entities for the Advancement of Technological Excellence, Japan Science and Technology Corporation, and Ultra-Thin Image Capturing Module, Science and Technology Incubation Program in Advanced Region, Japan Science and Technology Corporation.

\section{References}

1. W. Ford and M. S. Baum, Secure Electronic Commerce: Building the Infrastructure for Digital Signatures and Encryption (Prentice-Hall, Upper Saddle River, N.J., 1997).

2. A. K. Jain, L. Hong, and S. Pankanti, "Biometric identification," Commun. ACM 43, 91-98 (2000).

3. P. Tarasewich, "Wireless devices for mobile commerce: user interface design and usability," in Mobile Commerce: Technology, Theory, and Applications, B. E. Mennecke and T. J. Strader, ed. (Idea Group Publishing, Hershey, Penn., 2003), pp. 26-50.

4. L. O'Gorman, "Fingerprint verification," in Biometrics: Personal Identification in Networked Society, A. Jain, R. Bolle, and S. Pankanti, ed. (Kluwer Academic, Boston, Mass., 1999), pp. 43-64.

5. J. Tanida, T. Kumagai, K. Yamada, S. Miyatake, K. Ishida, T. Morimoto, N. Kondou, D. Miyazaki, and Y. Ichioka, "Thin observation module by bound optics (TOMBO): concept and experimental verification,” Appl. Opt. 40, 1806-1813 (2001).

6. J. Tanida, Y. Kitamura, K. Yamada, S. Miyatake, M. Miyamoto, T. Morimoto, Y. Masaki, N. Kondou, D. Miyazaki, and Y. Ichioka, "Compact image capturing system based on compound imaging and digital reconstruction," in Micro- and Nano-optics for Optical Interconnection and Information Processing, M. R. Taghizadeh, H. Thienpont, and G. E. Jabbonr, eds., Proc. SPIE 4455, 34-41 (2001).

7. D. I. Barnea and H. F. Silverman, "A class of algorithms for fast digital image registration," IEEE Trans. Comput. 21, 179-186 (1972).

8. Criminal Justice Information Services, "IAFIS Image quality specifications," in Electronic Fingerprint Transmission Specification, Int. Report. CJIS-RS-0010 (V7) Appendix F, (Criminal Justice Information Services Division, Washington, D.C., 1999). 